\title{
AN APPLICATION OF ROLL-INVARIANT POLARIMETRIC FEATURES FOR CROP CLASSIFICATION FROM MULTI-TEMPORAL RADARSAT-2 SAR DATA
}

\author{
M. Ustuner ${ }^{1, *}$, F. B. Sanli ${ }^{1}$, S. Abdikan ${ }^{2}$, M. T. Esetlili ${ }^{3}$, G. Bilgin ${ }^{4}$ \\ ${ }^{1}$ Dept. of Geomatic Engineering, Yildiz Technical University, Istanbul, Turkey - (mustuner, fbalik)@yildiz.edu.tr \\ ${ }^{2}$ Dept. of Geomatics Engineering, Bulent Ecevit University, Zonguldak, Turkey - sabdikan@ beun.edu.tr \\ ${ }^{3}$ Dept. of Soil Science and Plant Nutrition, Ege University, Izmir, Turkey - tolga.esetlili@ege.edu.tr \\ ${ }^{4}$ Dept. of Computer Engineering, Yildiz Technical University, Istanbul, Turkey - gbilgin@ yildiz.edu.tr
}

Commission I, WG I/3

KEY WORDS: Polarimetric SAR, Crop Classification, Multi-temporal Data, Agriculture, Synthetic Aperture Radar

\begin{abstract}
:
Crops are dynamically changing and time-critical in the growing season and therefore multitemporal earth observation data are needed for spatio-temporal monitoring of the crops. This study evaluates the impacts of classical roll-invariant polarimetric features such as entropy $(\mathrm{H})$, anisotropy (A), mean alpha angle $(\bar{\alpha})$ and total scattering power (SPAN) for the crop classification from multitemporal

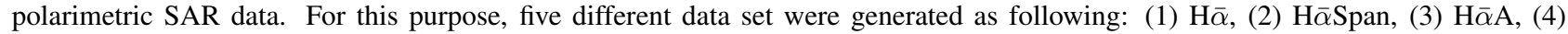
H $\bar{\alpha}$ ASpan and (5) coherency $[T]$ matrix. A time-series of four PolSAR data (Radarsat-2) were acquired as 13 June, 01 July, 31 July and 24 August in 2016 for the test site located in Konya, Turkey. The test site is covered with crops (maize, potato, summer wheat, sunflower, and alfalfa). For the classification of the data set, three different models were used as following: Support Vector Machines (SVMs), Random Forests (RFs) and Naive Bayes (NB). The experimental results highlight that H $\alpha$ ASpan (91.43\% for SVM, 92.25\% for RF and $90.55 \%$ for NB) outperformed all other data sets in terms of classification performance, which explicitly proves the significant contribution of SPAN for the discrimination of crops. Highest classification accuracy was obtained as $92.25 \%$ by RF and $\mathrm{H} \alpha \mathrm{ASpan}$ while lowest classification accuracy was obtained as $66.99 \%$ by NB and $\mathrm{H} \alpha$. This experimental study suggests that roll-invariant polarimetric features can be considered as the powerful polarimetric components for the crop classification. In addition, the findings prove the added benefits of PolSAR data investigation by means of crop classification.
\end{abstract}

\section{INTRODUCTION}

The growth of worldwide population and correspondingly the increasing demand of foods push the countries to develop low-cost and practical solutions for the applications of precision agriculture. For the sustainable agricultural practices in regional and national scale, the obtaining information about the crop acreage is of importance however owing to the very dynamic structure of crops in the growing season, the mapping of crops and obtaining information about the crop yield present a challenge. In comparison to other land cover types, the agricultural targets have the rapid and dynamic structural changes (i.e. phenological changes) within short time periods in the growing season (McNairn and Brisco, 2004, Bargiel, 2017). Synthetic Aperture Radar (SAR) sensors use the signals at microwave wavelengths and thereby have the weather/daylight in-dependent imaging capability. The radar systems, based on its unique calibration procedure, are powerful and advantageous for the information retrieval about land surface characteristics and changing surface dynamics since they benefit from the relationship between the target and back-scattered radar signal in terms of polarization, physics and illuminating geometry (Schmullius et al., 2015).

In this sense, spaceborne SAR sensors can be effectively used for time-critical agricultural applications and provide the crucial information for crop classification especially for multitemporal analysis. For the mono-temporal SAR data, two crops could have similar backscatter information however when the crops grow and move to the next stage in growing period, the backscatter of each crop type changes over the time and this leads to the better discrimination of the crops (McNairn and Brisco, 2004, McNairn and Shang, 2016). In several recent studies, the requirements and advantages of multi-temporal SAR images for successful crop classification and agricultural practices were stated (Jiao et al., 2014, Mirzaee et al., 2014, Larrañaga and Álvarez-Mozos, 2016, Bargiel, 2017, Dimov et al., 2017, Kussul et al., 2018, Woźniak et al., 2018). Jiao et al. (2014) investigated an object-oriented classification of multi-temporal RADARSAT-2 images for mapping and monitoring the crops. Mirzaee et al. (2014) tested the effects of phenological changes for the classification of agricultural fields using multitemporal TerraSAR-X images. Larrañaga and Álvarez-Mozos (2016) assessed the added value of quad-pol data in a multitemporal crop classification by using polarimetric features and object based random forest classification algorithm. Bargiel (2017) developed a new classification approach based on multitemporal data and crop phenology information by using the time-series of Sentinel-1 images. Dimov et al. (2017) compared the classification performance of time-series Sentinel-1 and Landsat- 8 data for the crop classification.Kussul et al. (2018) investigated the multitemporal SAR and optical data (Landsat-8, Sentinel-1 and Sentinel-2) for crop specific mapping. Woźniak et al. (2018) tested the dual-polarization polarimetric processing of time-series Sentinel-1 data on land cover classification. All these above studies stressed the requirements of multitemporal SAR data set for an adequate crop classification and consequently demonstrated the potential use of radar remote sensing for agricultural applications, especially crop classification and temporal monitoring.

${ }^{*}$ Corresponding author. 
Polarimetric SAR (PolSAR) sensors record the complete characteristics of scattering field (coherent channels and phase information) and enable of extracting the bio-geophysical parameters for the canopy structure of crops. In comparison to single or dualpolarized SAR data, PolSAR data provide more unique and significant details in terms of scattering mechanism for natural and man-made targets (Steele-Dunne et al., 2017). The differences among the scattering mechanisms of crop types enables the seperation of crops since this mechanism is related to crop phenology and crop type (Lopez-Sanchez et al., 2012, Hütt and Waldhoff, 2018). For better understanding and easier interpretation of the scattering mechanism of the targets, polarimetric features can be generated from the complex PolSAR data by using polarimetric decompositions. Polarimetric features were found to be quite helpful for land cover and crop type classification(McNairn and Brisco, 2004, Steele-Dunne et al., 2017). Hariharan et al.(2016) used the roll-invariant polarimetric features to classify urban areas using random forest (Hariharan et al., 2016). Tao et al. (2017) tested the potential use of roll-invariant and selected hidden polarimetric features in the rotation domain for land cover classification (Tao et al., 2017). Guo et al.(2018) investigated the rollinvariant polarimetric features (only entropy and mean alpha angle) for the crop classification by using multitemporal quad and dual-polarization SAR data (Guo et al., 2018). All these three aforementioned studies showed the importance of roll-invariant polarimetric features for classification purposes.

This study discusses the impacts of roll-invariant polarimetric features such as entropy, anisotropy, mean alpha angle, and total scattering power (SPAN) for the crop classification from multitemporal polarimetric SAR data. A time-series of four PolSAR data (single look complex full polarimetric Radarsat-2 with fine quad-polarization acquisition mode) were acquired for the study area located in Konya, Turkey. The H-A- $\bar{\alpha}$ (Cloude-Pottier) decomposition decomposition were used to obtain the roll-invariant polarimetric features (entropy, anisotropy and mean alpha angle).

The remainder of the paper is organized as follows. The data description and the details of data processing and image classification are given Section 2. In Section 3, the experimental results are presented and discussed. In the end, Section 4 provides some final conclusions and directions for future work.

\section{METHODOLOGY}

\subsection{Polarimetric Features}

Classical roll-invariant polarimetric features which are not dependent of target orientations can extracted from the eigenvalue and eigenvector based decomposition of the coherency matrix by using $\mathrm{H}-\mathrm{A}-\bar{\alpha}$ decomposition method. From this decomposition, the coherency matrix are formed as following:

$$
\mathbf{T}=U\left[\begin{array}{ccc}
\lambda_{1} & 0 & 0 \\
0 & \lambda_{2} & 0 \\
0 & 0 & \lambda_{3}
\end{array}\right] U^{H}, \lambda_{1} \geqslant \lambda_{2} \geqslant \lambda_{3}
$$

where $\lambda_{1}, \lambda_{2}$ and $\lambda_{3}$ are the eigenvalues and $U$ represent the decomposed eigenvectors. And the roll-invariant polarimetric features extracted from the $\mathrm{H}-\mathrm{A}-\bar{\alpha}$ decomposition are defined as follows (Cloude and Pottier, 1996, Lee and Pottier, 2009):

$$
\mathbf{H}=-\sum_{i=1}^{3} P_{i} \log _{3}\left(P_{i}\right)
$$

$$
\begin{gathered}
\bar{\alpha}=\sum_{i=1}^{3} P_{i} \alpha_{i} \\
\mathbf{A}=\frac{\lambda_{2}-\lambda_{3}}{\lambda_{2}+\lambda_{3}}
\end{gathered}
$$

where

$$
P_{i}=\frac{\lambda_{i}}{\lambda_{2}+\lambda_{2}+\lambda_{3}}
$$

SPAN is considered as the total backscattering power and preferred in PolSAR image analysis since the speckle noise is relatively reduced compared to single channels of data (Wang et al., 2017). The SPAN is formulated as follows:

$$
\mathbf{S P A N}=\sum_{i=1}^{3} \lambda_{i}
$$

An example of the roll-invariant polarimetric features for monotemporal data (for July 31, 2016) is shown in Figure 1
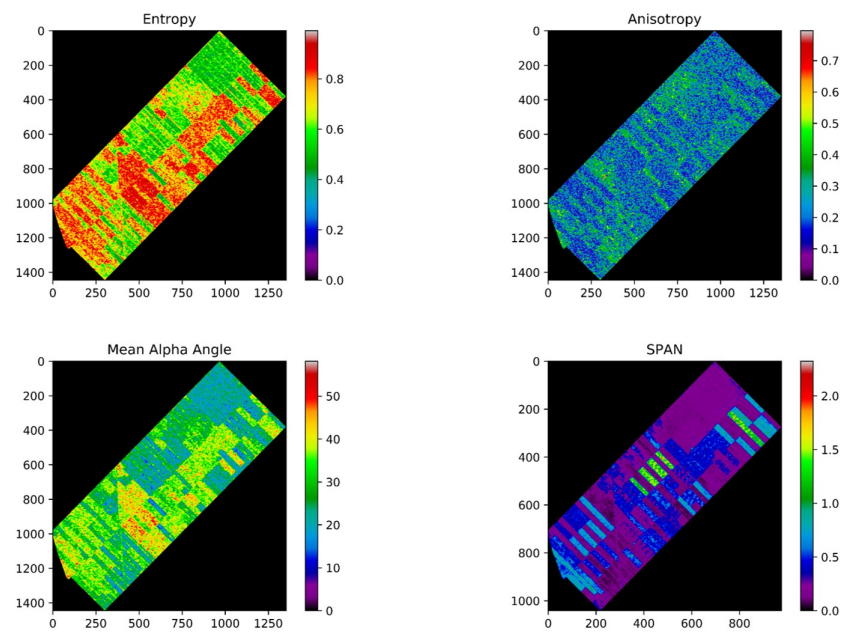

Figure 1. Roll-invariant polarimetric features

\subsection{Data and Preprocessing}

A time-series of four Radarsat-2 acquisitions (single look complex full polarimetric Radarsat-2 data with fine quad-polarization acquisition mode) were acquired as June 13, July 01, July 31 and August 24 of the year 2016 for the study area located in Konya basin, Turkey. The image characteristics are presented in Table 1.

\begin{tabular}{|l|c|}
\hline Specifications & Description \\
\hline Sensor & Radarsat-2 \\
Wavelength & C Band / 5.6 cm \\
Imaging mode & Fine quad-pol \\
Orbit & Descending \\
Incidence Angle & $40^{\circ}$ \\
Resolution (Rg x Az) & $4.7 \times 5.1(\mathrm{~m})$ \\
Polarization & Quad-pol \\
\hline
\end{tabular}

Table 1. Image characteristics (Morena et al., 2004) 
The pre-processing of the SAR data is important to extract relevant information from the data for a successful crop classification. The following processing steps were applied to the PolSAR data to extract the entropy, mean alpha angle and anisotropy: First, the data were imported into ESA (European Space Agency) SNAP (The Sentinel Application Platform) toolbox and calibrated. Following this step, the Sinclair scattering matrices were converted to the coherency matrix for further polarimetric processing. After this step, $\mathrm{H}-\mathrm{A}-\bar{\alpha}$ decomposition method was applied to the data to extract roll-invariant polarimetric features except SPAN. During the polarimetric decomposition process, window size was determined as $9 \times 9$. And then the geometric terrain correction was applied to the imagery using the Range Doppler orthorectification method. During the terrain correction, the Shuttle Radar Topography Mission (SRTM) data (1 arc second, approximately $30-\mathrm{m}$ resolution) was automatically downloaded and used. Then, the data were resampled to 10 meter pixel resolution using bilinear interpolation.As the last step of the data preprocessing, the data were scaled to decibels $[\mathrm{dB}]$ and exported in GeoTIFF format. For extracting the SPAN band, almost the same pre-processing chain was applied except matrix generation and polarimetric decomposition process. The data were calibrated and then SPAN was generated through SNAP toolbox. The following step were the terrain correction, scaling the data into $\mathrm{dB}$ and exporting the data in GeoTIFF format. Figure 2 shows the pre-processing chain for each acquisition.

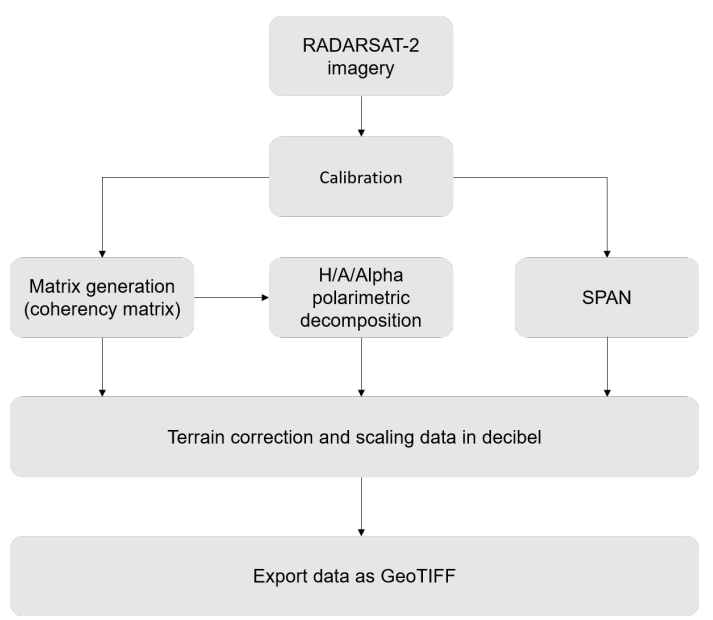

Figure 2. Workflow diagram for pre-processing chain

After extraction of the roll-invariant polarimetric features for each acquisition time, the time-series stack data were created for the roll-invariant polarimetric features. The number of bands/features for our data set in monotemporal as well as time-series stack data can be seen in Table 2. All pre-processing steps were implemented using the open source SNAP toolbox v6.0.1.

\begin{tabular}{|l|c|c|}
\hline Data set & \multicolumn{2}{|c|}{ Number of bands } \\
\hline & Monotemporal & Time-Series Stack \\
$\mathrm{H} \bar{\alpha}$ & 2 & 8 \\
$\mathrm{H} \bar{\alpha}$ Span & 3 & 12 \\
$\mathrm{H} \bar{\alpha} \mathrm{A}$ & 3 & 12 \\
$\mathrm{H} \bar{\alpha} \mathrm{ASpan}$ & 4 & 16 \\
coherency $[\mathrm{T}]$ matrix & 6 & 24 \\
\hline
\end{tabular}

Table 2. Number of features

\subsection{Study Area and Ground Truth Information}

The study area (Figure 3) is located in Konya basin, Turkey and only covered with agricultural lands. Semi-arid climate is dominated in the region. The study area covers with the crop types which are as following: alfalfa, maize, potato, summer wheat, and sunflower for the vegetation season of 2016 summer season. In-situ data were collected using a handheld GPS at the acquisition dates of each satellite imagery and were recorded to be used in the crop classification and accuracy assessment. The number of pixels for training and testing data are shown in Table 3.

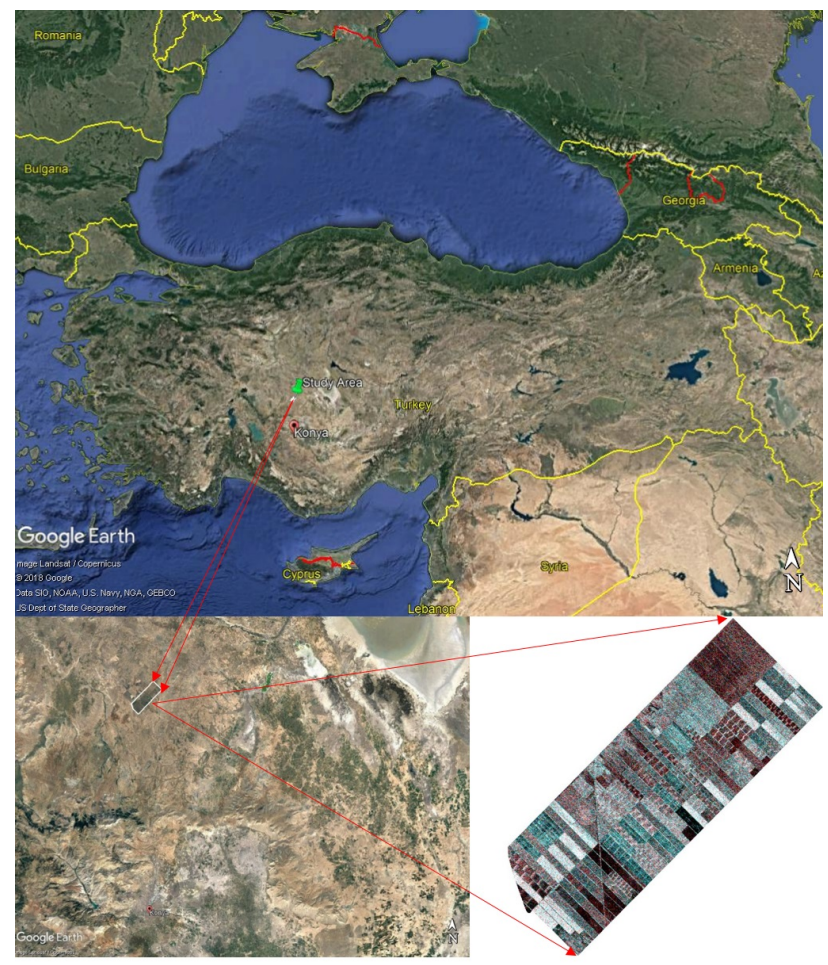

Figure 3. Study area

\begin{tabular}{|l|l|l|}
\hline \multirow{2}{*}{ Class } & \multicolumn{2}{|l|}{ Number of Pixels } \\
\cline { 2 - 3 } & Training & Testing \\
\hline Alfalfa & 1918 & 3542 \\
Maize & 5581 & 14217 \\
Potato & 2275 & 10604 \\
Wheat & 3524 & 6338 \\
Sunflower & 3729 & 8915 \\
\hline
\end{tabular}

Table 3. Training and Testing Data

Furthermore, their spatial distribution of the training and testing data can be shown in Figure 4.

\subsection{Image Classification}

With the major advances in the machine learning and computer vision, there has been an increasing trend in machine learning algorithms for the operational use in the fields of remote sensing and pattern recognition, especially for the classification purposes. Image classification can still be considered as one of the powerful methods to extract information from the remotely-sensed images however are of some uncertainties such as the redundant (unnecessarily repeated) information among the spectral bands, imbalance of training samples in classes and the adaptation problem of training samples with imagery and implemented model 


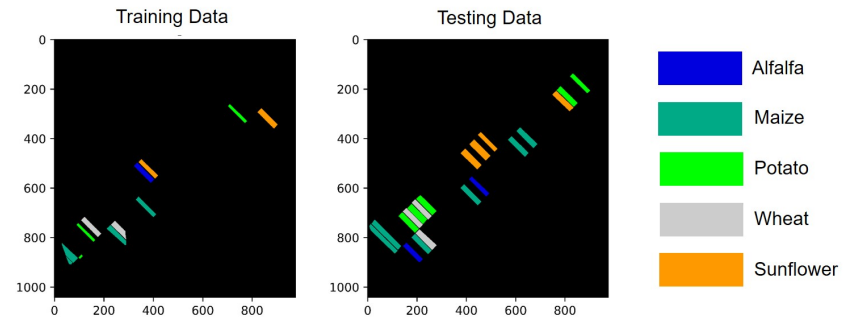

Figure 4. Spatial distribution of training and testing data

(Ghamisi et al., 2017). In this study, three different classification model were used as following: Support Vector Machines (SVMs), Random Forests (RFs) and Naive Bayes (NB). The classification models implemented in this study were briefly outlined in the following paragraph.

Naive Bayes classifier is one of the probabilistic classifiers in machine learning and uses Bayes theorem to predict the class of unknown data. This method calculates the posterior probability for each class and assign the particular pixel to the class with the highest posterior probability (Cánovas-García and Alonso-Sarría, 2015). Support Vector Machines are one of the kernel-based learning methods in machine learning and have been successfully and widely implemented in remote sensing and pattern recognition for classification and regression problems. SVMs aim to define the optimal hyperplane separating the two classes with the maximum margin width. In case of linearly inseparable two classes, it use the kernel trick (the non-linear version of SVMs) to map the data into higher dimensional space (Melgani and Bruzzone, 2004, Kavzoglu and Colkesen, 2009). Random forests are one of the ensemble (multiple) classifiers and use many decision trees to predict the final class for each unknown pixel. The final decision boundary is constructed by the majority voting of all the individual trees (Pal, 2005, Gislason et al., 2006).

All classifications were performed using the open-source Scikitlearn module in Python v3.6.4 (Pedregosa et al., 2011).

\section{RESULTS AND DISCUSSION}

In this section, classification accuracies of the five different data sets for each model and the class based accuracies via F1-score are presented. The classification accuracies of the models for each data set are reported in Table 4.

\begin{tabular}{|l|l|l|l|}
\hline \multirow{2}{*}{ Feature } & \multicolumn{3}{|c|}{ Overall Accuracy (\%) } \\
\cline { 2 - 4 } & SVM & RF & NB \\
\hline$[T]$ matrix & 77.35 & 76.13 & 71.57 \\
H- $\bar{\alpha}$ & 75.01 & 72.83 & 66.99 \\
H- $\bar{\alpha}$-SPAN & 89.19 & 92.06 & 89.85 \\
H- $-\bar{\alpha}-\mathrm{A}$ & 75.08 & 76.51 & 72.20 \\
H- $\bar{\alpha}$-A-SPAN & 91.43 & 92.25 & 90.55 \\
\hline
\end{tabular}

Table 4. Classification Accuracy (Overall accuracy)

The experimental results highlight that the classification of $\mathrm{H}-$ $\bar{\alpha}$-SPAN $(89.19 \%$ for SVM, $92.06 \%$ for RF and $89.85 \%$ for NB) and $\mathrm{H}-\bar{\alpha}$-A-SPAN (91.43\% for SVM, $92.25 \%$ for RF and $90.55 \%$ for NB) outperformed all other data sets in terms of classification performance, which also explicitly prove the significant contribution of SPAN parameter for the discrimination of crops. The incorporation of SPAN parameter into $\mathrm{H}-\bar{\alpha}$ data set increased the overall classification accuracy as $14.18 \%, 19.23 \%$ and $22.87 \%$ for SVM, RF and NB, respectively. Highest classification accuracy was obtained as $92.25 \%$ by $\mathrm{RF}$ and $\mathrm{H}-\bar{\alpha}$-A-SPAN while lowest classification accuracy was obtained as $66.99 \%$ by NB and $\mathrm{H}-\bar{\alpha}$ as seen in Table 4. The classification maps of the $\mathrm{H}-\bar{\alpha}$ and $\mathrm{H}-\bar{\alpha}$-A-SPAN data sets are shown in Figure 5. The main reason of choosing this two data sets is that highest and lowest classification accuracies for per model were obtained from these SAR data sets.
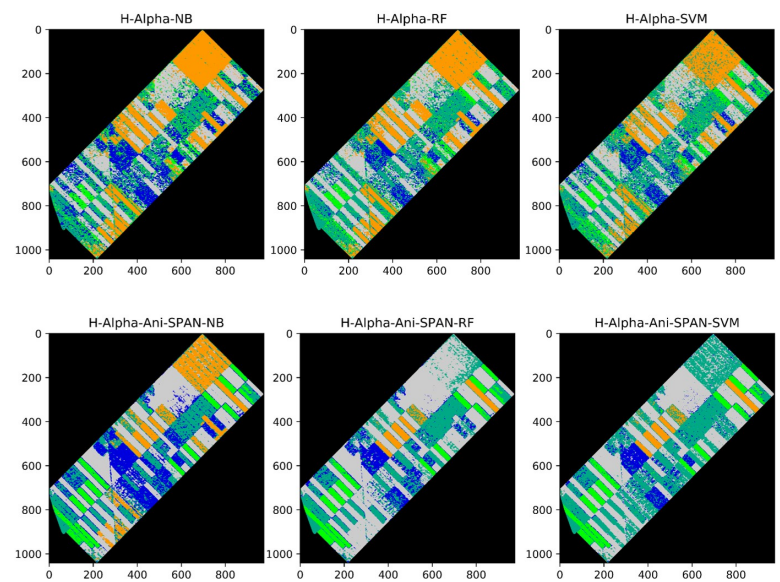

5

Figure 5. Classification maps for the study area

F1-score is defined in equation (7) as following.

$$
\mathbf{F 1}=\frac{2 a}{2 a+b+c}
$$

\begin{tabular}{|l|l|l|l|}
\hline & \multicolumn{3}{|c|}{ True } \\
\hline \multirow{3}{*}{ Predicted } & & Positive & Negative \\
\cline { 2 - 4 } & Positive & True Positive (a) & False Positive (b) \\
\cline { 2 - 4 } & Negative & False Negative (c) & True Negative (d) \\
\hline
\end{tabular}

Table 5. Confusion Matrix

The class based accuracies were compared based on F1-score values which were reported for each classification model in Table 6 .

\begin{tabular}{|l|l|c|c|c|c|c|}
\hline Model & Class & $\mathbf{1}$ & $\mathbf{2}$ & $\mathbf{3}$ & $\mathbf{4}$ & $\mathbf{5}$ \\
\hline \multirow{5}{*}{ SVM } & Alfalfa & 0.26 & 0.24 & 0.26 & 0.28 & 0.33 \\
& Maize & 0.75 & 0.71 & 0.88 & 0.72 & 0.91 \\
& Potato & 0.83 & 0.66 & 0.93 & 0.66 & 0.97 \\
& Sunflower & 0.80 & 0.98 & 0.99 & 0.97 & 1.00 \\
& Wheat & 0.95 & 0.93 & 0.94 & 0.93 & 0.96 \\
\hline \multirow{5}{*}{ RF } & Alfalfa & 0.20 & 0.22 & 0.42 & 0.26 & 0.43 \\
& Maize & 0.74 & 0.69 & 0.91 & 0.74 & 0.92 \\
& Potato & 0.80 & 0.55 & 0.97 & 0.64 & 0.97 \\
& Sunflower & 0.79 & 0.99 & 1.00 & 0.99 & 1.00 \\
& Wheat & 0.95 & 0.94 & 0.97 & 0.95 & 0.97 \\
\hline \multirow{5}{*}{ NB } & Alfalfa & 0.25 & 0.25 & 0.44 & 0.25 & 0.45 \\
& Maize & 0.66 & 0.62 & 0.87 & 0.69 & 0.88 \\
& Potato & 0.80 & 0.50 & 0.96 & 0.62 & 0.97 \\
& Sunflower & 0.75 & 0.99 & 1.00 & 0.99 & 1.00 \\
& Wheat & 0.94 & 0.94 & 0.98 & 0.94 & 0.98 \\
\hline
\end{tabular}

Table 6. Class accuracies via F-1 score

In Table 6 , the numbers of $(\mathbf{1}, \mathbf{2}, \mathbf{3}, \mathbf{4}, \mathbf{5})$ represent the $[T]$ matrix, $\mathrm{H}-\bar{\alpha}, \mathrm{H}-\bar{\alpha}-\mathrm{SPAN}, \mathrm{H}-\bar{\alpha}-\mathrm{A}$ and $\mathrm{H}-\bar{\alpha}-\mathrm{A}-\mathrm{SPAN}$, respectively. 
When F-score values for per class were examined, it is reported that Alfalfa was the most-confusing class among crop types. The maximum separation ratio for Alfalfa was obtained with NB and $\mathrm{H}-\bar{\alpha}$-A-SPAN however this value is still less than 0.50. For maize class, the F-score values were obtained as 0.62 and 0.69 for $\mathrm{H}-\bar{\alpha}$ and by NB and RFs. With the incorporation of SPAN into the $\mathrm{H}-$ $\bar{\alpha}$, the class accuracy values of maize were obtained as 0.88 and 0.92 for NB and RFs, respectively. The similar increase trend was also reported for potato class. The F-score values of the sunflower class were obtained as 1.00 with all classification models for the data set of and $\mathrm{H}-\bar{\alpha}$-A-SPAN.This is the maximum F-score value among other classes. For all data sets, the F-score values are higher than 0.75 for sunflower class. For wheat class, the F-score values were obtained higher than 0.90 for all data set and implemented models. The highest value of F-score was obtained as 1.00 for the sunflower class while the lowest value was obtained 0.20 for the Alfalfa class.

\section{CONCLUSIONS}

In this study, the impacts of classical roll-invariant polarimetric features for the crop classification from multi-temporal polarimetric SAR data were investigated. To explore the impacts of polarimetric features, five different data sets were created and used as input data for classification. The incorporation of SPAN parameter into $\mathrm{H}-\bar{\alpha}$ data set increased the overall classification accuracy as $14.18 \%, 19.23 \%$ and $22.87 \%$ for SVM, RF and NB, respectively. These increases explicitly demonstrate the significant contribution of SPAN parameter for the discrimination of crops. SVMs outperformed other methods for the classification of $[T]$ matrix because of the main capability of the SVMs in handling high-dimensional data with a limited number of training samples. RFs received highest classification accuracies for all data sets except $\mathrm{H}-\bar{\alpha}$ and $[T]$ matrix. The results emphasize that the polarimetric features have to be extensively analysed and investigated in terms of the relationship between the changes in the phenological pattern of the crops and the polarimetric scattering. This experimental study suggests that roll-invariant polarimetric features can be considered as the powerful polarimetric components for the crop classification from multi-temporal polarimetric SAR. In addition, the findings prove the added benefits of PolSAR data investigation by means of crop classification. Our future research will address the extensive analysis and interpretation of the polarimetric features obtained from target decompositions for crop classification from multi-temporal SAR data.

\section{ACKNOWLEDGEMENTS}

The data used in this research were supported by TAGEM: General Directorate of Agricultural Research and Policies, Turkey (Project No: TAGEM/TSKAD/14/A13/P05/03). The authors gratefully acknowledge the support from TAGEM. The first author of the paper was supported by TUBITAK (The Scientific and Technological Research Council of Turkey) 2214/A International Doctoral Research Fellowship Programme. The experiments were held while the first author visited the Remote Sensing Section of Geography Department at Friedrich-Schiller University Jena, Germany as a guest researcher. The first author would like to thank TUBITAK (Grant No \#1059B141700579) for their support and also to all members of Geography Department of FriedrichSchiller University Jena, Germany for their hospitality.

\section{REFERENCES}

Bargiel, D., 2017. A new method for crop classification combining time series of radar images and crop phenology information. Remote Sensing of Environment 198, pp. 369 - 383.

Cánovas-García, F. and Alonso-Sarría, F., 2015. Optimal combination of classification algorithms and feature ranking methods for object-based classification of submeter resolution z/i-imaging dmc imagery. Remote Sensing 7(4), pp. 4651-4677.

Cloude, S. R. and Pottier, E., 1996. A review of target decomposition theorems in radar polarimetry. IEEE Transactions on Geoscience and Remote Sensing 34(2), pp. 498-518.

Dimov, D., Löw, F., Ibrakhimov, M., Stulina, G. and Conrad, C., 2017. Sar and optical time series for crop classification. In: 2017 IEEE International Geoscience and Remote Sensing Symposium (IGARSS), pp. 811-814.

Ghamisi, P., Plaza, J., Chen, Y., Li, J. and Plaza, A. J., 2017. Advanced spectral classifiers for hyperspectral images: A review. IEEE Geoscience and Remote Sensing Magazine 5(1), pp. 8-32.

Gislason, P. O., Benediktsson, J. A. and Sveinsson, J. R., 2006. Random forests for land cover classification. Pattern Recognition Letters 27(4), pp. 294 - 300. Pattern Recognition in Remote Sensing (PRRS 2004).

Guo, J., Wei, P. L., Liu, J., Jin, B., Su, B. F. and Zhou, Z. S., 2018. Crop classification based on differential characteristics of $H / \alpha$ scattering parameters for multitemporal quad- and dualpolarization sar images. IEEE Transactions on Geoscience and Remote Sensing pp. 1-13.

Hariharan, S., Tirodkar, S. and Bhattacharya, A., 2016. Polarimetric sar decomposition parameter subset selection and their optimal dynamic range evaluation for urban area classification using random forest. International Journal of Applied Earth Observation and Geoinformation 44, pp. $144-158$.

Hütt, C. and Waldhoff, G., 2018. Multi-data approach for crop classification using multitemporal, dual-polarimetric terrasar-x data, and official geodata. European Journal of Remote Sensing 51(1), pp. 62-74.

Jiao, X., Kovacs, J. M., Shang, J., McNairn, H., Walters, D., Ma, B. and Geng, X., 2014. Object-oriented crop mapping and monitoring using multi-temporal polarimetric radarsat-2 data. ISPRS Journal of Photogrammetry and Remote Sensing 96, pp. 38 - 46.

Kavzoglu, T. and Colkesen, I., 2009. A kernel functions analysis for support vector machines for land cover classification. International Journal of Applied Earth Observation and Geoinformation 11(5), pp. $352-359$.

Kussul, N., Mykola, L., Shelestov, A. and Skakun, S., 2018. Crop inventory at regional scale in ukraine: developing in season and end of season crop maps with multi-temporal optical and sar satellite imagery. European Journal of Remote Sensing 51(1), pp. 627-636.

Larrañaga, A. and Álvarez-Mozos, J., 2016. On the added value of quad-pol data in a multi-temporal crop classification framework based on radarsat-2 imagery. Remote Sensing.

Lee, J.-S. and Pottier, E., 2009. Polarimetric radar imaging: from basics to applications. CRC press. 
Lopez-Sanchez, J. M., Cloude, S. R. and Ballester-Berman, J. D., 2012. Rice phenology monitoring by means of sar polarimetry at $\mathrm{x}$-band. IEEE Transactions on Geoscience and Remote Sensing 50(7), pp. 2695-2709.

McNairn, H. and Brisco, B., 2004. The application of c-band polarimetric sar for agriculture: a review. Canadian Journal of Remote Sensing 30(3), pp. 525-542.

McNairn, H. and Shang, J., 2016. A Review of Multitemporal Synthetic Aperture Radar (SAR) for Crop Monitoring. Springer International Publishing, Cham, pp. 317-340.

Melgani, F. and Bruzzone, L., 2004. Classification of hyperspectral remote sensing images with support vector machines. IEEE Transactions on Geoscience and Remote Sensing 42(8), pp. $1778-1790$.

Mirzaee, S., Motagh, M., Arefi, H. and Nooryazdan, M., 2014. Classification of agricultural fields using time series of dual polarimetry terrasar-x images. ISPRS - International Archives of the Photogrammetry, Remote Sensing and Spatial Information Sciences XL-2/W3, pp. 191-196.

Morena, L. C., James, K. V. and Beck, J., 2004. An introduction to the radarsat-2 mission. Canadian Journal of Remote Sensing 30(3), pp. 221-234.

Pal, M., 2005. Random forest classifier for remote sensing classification. International Journal of Remote Sensing 26(1), pp. 217 222.

Pedregosa, F., Varoquaux, G., Gramfort, A., Michel, V., Thirion, B., Grisel, O., Blondel, M., Prettenhofer, P., Weiss, R., Dubourg, V., Vanderplas, J., Passos, A., Cournapeau, D., Brucher, M., Perrot, M. and Duchesnay, E., 2011. Scikit-learn: Machine learning in Python. Journal of Machine Learning Research 12, pp. 28252830 .

Schmullius, C., Thiel, C., Pathe, C. and Santoro, M., 2015. Radar Time Series for Land Cover and Forest Mapping. Springer International Publishing, Cham, pp. 323-356.

Steele-Dunne, S. C., McNairn, H., Monsivais-Huertero, A., Judge, J., Liu, P. W. and Papathanassiou, K., 2017. Radar remote sensing of agricultural canopies: A review. IEEE Journal of Selected Topics in Applied Earth Observations and Remote Sensing 10(5), pp. 2249-2273.

Tao, C., Chen, S., Li, Y. and Xiao, S., 2017. Polsar land cover classification based on roll-invariant and selected hidden polarimetric features in the rotation domain. Remote Sensing.

Wang, X., Cao, Z., Ding, Y. and Feng, J., 2017. Composite kernel method for polsar image classification based on polarimetricspatial information. Applied Sciences.

Woźniak, E., Kofman, W., Lewinski, S., Wajer, P., Rybicki, M., Aleksandrowicz, S. and Włodarkiewicz, A., 2018. Multitemporal polarimetry in land-cover classification. International Journal of Remote Sensing 0(0), pp. 1-18. 